\title{
Risk Factors for Surgical Site Infection Following Total Joint Arthroplasty
}

\author{
Mohammad Rasouli, MD \\ Rothman Institute of Orthopaedics at the Thomas Jefferson University Hospital \\ Camilo Restrepo, MD \\ Rothman Institute of Orthopaedics at the Thomas Jefferson University Hospital \\ Mitchell Maltenfort, PhD \\ Rothman Institute of Orthopaedics at the Thomas Jefferson University Hospital \\ James J. Purtill, MD \\ Rothman Institute of Orthopaedics at the Thomas Jefferson University Hospital \\ Javad Parvizi, MD \\ Rothman Institute Thomas Jefferson University \\ Follow this and additional works at: https://jdc.jefferson.edu/rothinsposters \\ Part of the Orthopedics Commons \\ Let us know how access to this document benefits you
}

\section{Recommended Citation}

Rasouli, MD, Mohammad; Restrepo, MD, Camilo; Maltenfort, PhD, Mitchell; Purtill, MD, James J.; and Parvizi, MD, Javad, "Risk Factors for Surgical Site Infection Following Total Joint Arthroplasty" (2014). Rothman Institute Conference Posters. Paper 6.

https://jdc.jefferson.edu/rothinsposters/6

This Article is brought to you for free and open access by the Jefferson Digital Commons. The Jefferson Digital Commons is a service of Thomas Jefferson University's Center for Teaching and Learning (CTL). The Commons is a showcase for Jefferson books and journals, peer-reviewed scholarly publications, unique historical collections from the University archives, and teaching tools. The Jefferson Digital Commons allows researchers and interested readers anywhere in the world to learn about and keep up to date with Jefferson scholarship. This article has been accepted for inclusion in Rothman Institute Conference Posters by an authorized administrator of the Jefferson Digital Commons. For more information, please contact: JeffersonDigitalCommons@jefferson.edu. 


\section{INTRODUCTION}

Surgical Site Infection (SSI) after total joint arthroplasty (TJA) is a rare but devastating complication'. In spite of improvement in the prevention of SSI, these infections are still a signifcant cause of morbidity in surgical patients. Management of Hospital Acquired infections (HA) including SSI poses a huge economic burden on healthcare. As part of the mission to reduce the
burden of HAl, the Centers for Disease Control and Prevention (CDC) has issued guidelines for he prevention of SSI that are currently being updated4. In addition, CDC requires all hospitals to It is believed that identifial Healthcare Safety Network (NHSN) surveillance program.

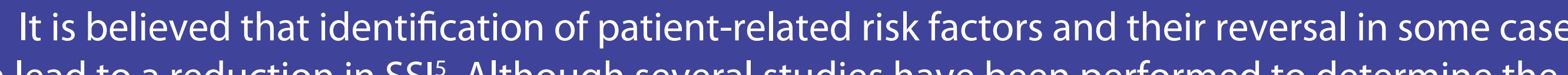
isk factors of SSI following TJA'1.6 risk factors for SSI as defined by the CDC and required to be reported has not been fully evaluated. The objective of this case-control study was to determine the patient-related risk factors for SSI following primary and revision TJA using our institutional database on TJA and the data generated by the NHSN surveillance.

\section{MATERIALS AND METHODS}

Upon approval of the Institutional Review Board, 6111 primary and revision TJAs performed between April 2010 and June 2012 were identified. SSI cases based on the CDC definition were captured by infection control nurses in one of the 4 following ways: 1) An alert is sent to surveillance center from microbiology whenever a culture from a patient isolates an organism, 2) An or affliated hospitals, 3) an automatic check is in place to scan the operating room schedules and detect the names of patients who had arthroplasty within the preceding year, and 4) our institua is int formed at another institution were excluded and all cases were followed up for one year regarding development of SSI.

nootstrap resampling were used respectively to create and validate the model for predictors of SSI. The predictive power of the model was estimated by the area under the curve (AUC) in receiver operating characteristic plots.

\section{RESULTS}

During the study interval 6,111 TJA were performed in 3,414 women and 2,697 men with a

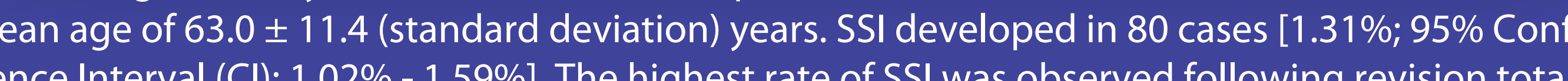
knee arthroplasty (TKA) at $4.57 \%(95 \% \mathrm{Cl}: 2.31 \%-6.83 \%)$ followed by revision total hip arthroplasty (THA) at $1.94 \%(95 \%$ Cl: $0.75 \%-3.13 \%)$

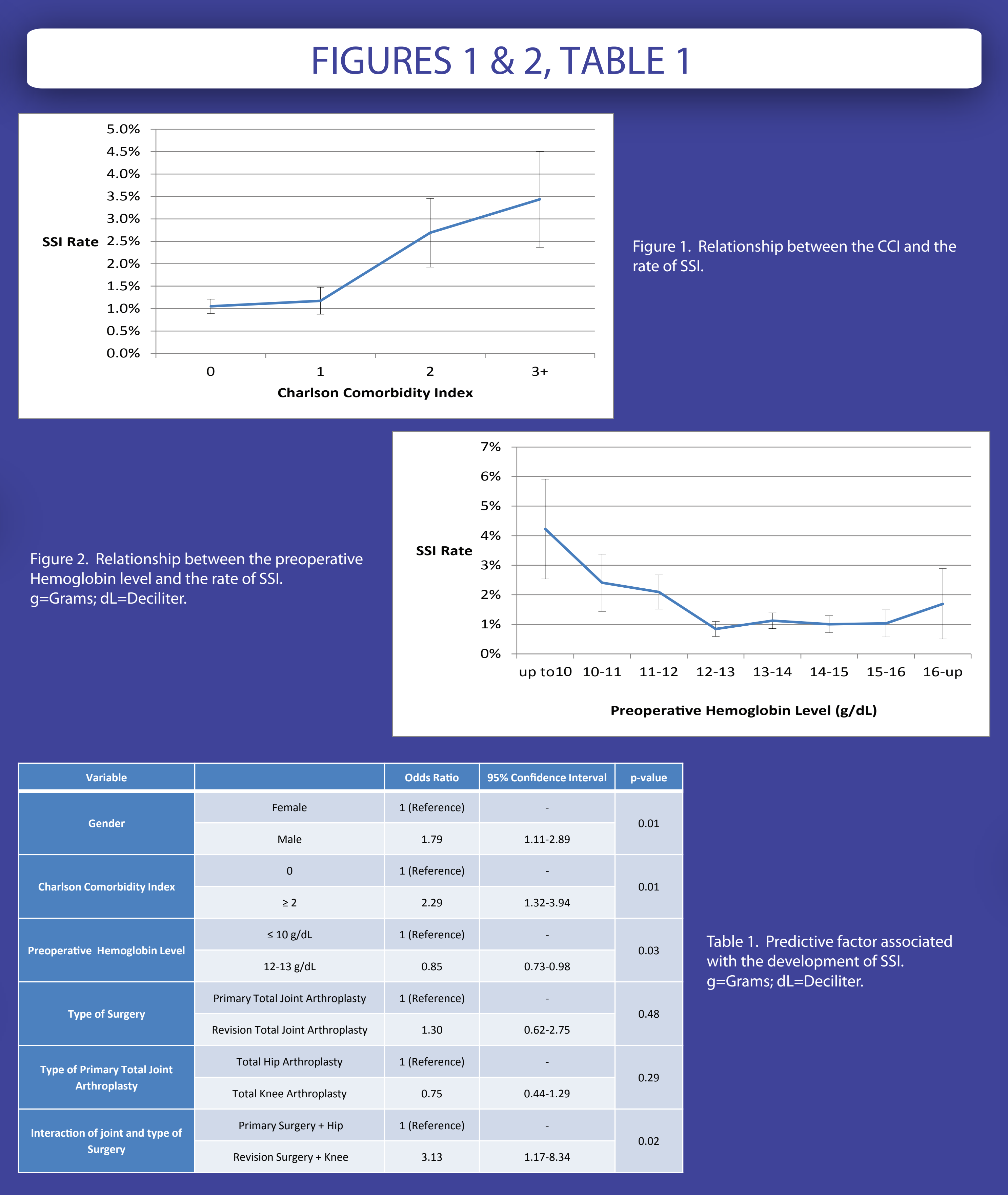

RESULTS As Figure 1 demonstrates, the rate of SSI increased in patients with a higher Charlson Comor
bidity Index (CCI). The highest rate of SSI at $4.23 \%(95 \%$ Cl: $0.92 \%-7.53 \%)$ was found in patients with a preoperative hemoglobin level of $\leq 10 \mathrm{~g} / \mathrm{dL}$ (Figure 2).

Multivariate logistic regression indicated that higher CCI male gender, revision TKA, and lower preoperative hemoglobin level are independent predictors of SSI (Table 1). The AUC of the model Was found to be 0.709 without correction and 0.678 after bootstrap correction for model optimism 200 bootstrap samples). These values indicate that the model has fair predictive power.

\section{DISCUSSION}

The study has some limitations. Despite the availability of a comprehensive database, this

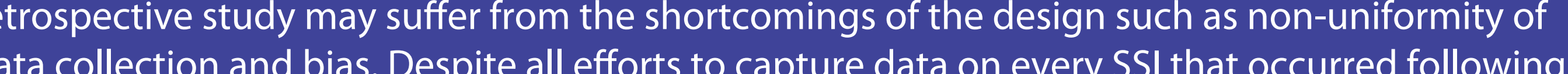
TJA in this cohort it is possible that some cases of SSI that were seen and treated on an outpatient basis may have been missed. However, we feel the latter is unlikely, as ordering of any cultures would have led to the notification of the infection surveillance center. We included only variables with the highest probability of affecting SSI in the model to avoid the potential negative effect that entering too many variables in the presence of a small number of events could have on the model. This might be considered as one of the limitations of this study. However, we used various

In conclusion, this study, comprising of a relatively large cohort of patients receiving TJA at a
ats

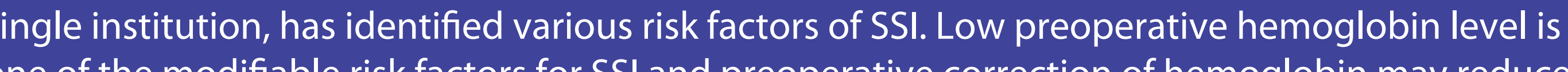
one of the modifiable risk factors for SSI and preoperative correction of hemoglobin may reduce lobin with not correcting the preoperative hemoglobin level is recommended.

REFERENCES

Namba RS, Inacio MC, Paxton EW. Risk factors associated with deep surgical site infections affer primary total kne arthroplasty: an analysis of 56,216 knees.J Jone Joint Surg Am. 2013;95(9):775-82.
Owens CD, Stoessel K. Surgical site infections: epidemiology, microbiology and prevention. J Hosp Infect. 2008;70 (Suppl 2):3-10.
Nero DC, Lipp M, Callahan MA. The financial impact of hospital-acquired conditions.J J Health Care Finance.

Mangram A, Horan TC, Pearson ML, Silver LC, Jarvis WR. Guideline for Prevention of Surgical S Site Infection, 1999.
Centers for Disease Control and Prevention (CDC) Hospitial Infection Control Practices Advisory Committee. Am J Infect Control. 1999:27(2):97:112.

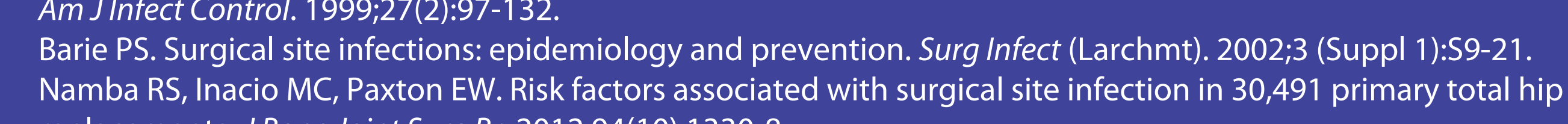

